\title{
In-vitro resistance to azoles associated with mitochondrial DNA deficiency in Candida glabrata
}

\author{
ALAIN DEFONTAINE, JEAN-PHILIPPE BOUCHARA*, PHILIPPE DECLERK*, CLAIRE \\ PLANCHENAULT*, DOMINIQUE CHABASSE* and JEAN-NOEL HALLET
}

Laboratoire de Biotechnologie, UPRES 2161 Biocatalyse, 2 rue de la Houssinière, 44322 Nantes Cedex 03 and * Groupe d'Etude des Interactions Hôtes-Parasite, Laboratoire de Parasitologie-Mycologie, Centre Hospitalier Universitaire, 4 rue Larrey, Angers Cedex 01, France

\begin{abstract}
A commercially available disk diffusion procedure was used in a large-scale study to evaluate the susceptibility of a wide range of Candida isolates to polyenes and azoles. With almost all isolates of $C$. glabrata resistant colonies were present within the inhibition zones for the azole compounds fluconazole, ketoconazole and miconazole, and less frequently for isoconazole, econazole and clotrimazole. Ten randomly selected isolates were cloned by limiting dilution and the susceptibility of the resulting strains to polyenes and azoles was determined. All strains presented a similar susceptibility pattern with sensitivity to polyenes and the presence of resistant colonies for all azole compounds except tioconazole. For each strain and each antifungal agent, one of these resistant colonies was subcultured and studied for antifungal susceptibility. All these colonies showed similar properties regardless of which antifungal agent allowed their selection, with increased sensitivity to polyenes and cross-resistance to the azole compounds except tioconazole. Similar results were obtained on Shadomy's modified medium and on synthetic medium. Likewise, determination of MICs by the Etest method confirmed the resistance to fluconazole. Comparative growth studies revealed a respiratory deficiency in the mutants caused by mitochondrial DNA (mtDNA) deletions. In addition, 'petite' mutants were obtained from a wild-type strain by exposure to ethidium bromide, and these respiratory mutants were shown to be resistant to azoles. These results demonstrate the relationship between mtDNA deficiency and resistance to azoles, and provide an interesting model to study the mechanisms of action of these antifungal agents.
\end{abstract}

\section{Introduction}

During the past two decades, the development of antibiotic treatments, the widespread use of immunosuppressive therapy and the emergence of AIDS have led to a marked increase in the prevalence of candidosis. Moreover, as a consequence of the resulting extensive use of antifungal agents, a shift in the nature of the infecting organisms has been reported [1,2]. Candida albicans remains the most frequent causative agent, but infections due to other yeast species initially considered as non-pathogens, such as $C$. kruse $i$ and $C$. glabrata, are reported increasingly. C. glabrata, the second commonest species in vaginal candidosis and candiduria, has become the second most common species in candidaemia

Received 24 July 1998; revised version accepted 6 Nov. 1998.

Corresponding author: Dr A. Defontaine.

(e-mail: defontaine@nat.sut.sciences.univ-nantes.Fr). in some centres, its frequency in this situation increasing from $2 \%$ in 1987 to $26 \%$ in 1992 [3].

To treat these infections, several potent antifungal imidazoles and triazoles have been developed. Because of its pharmacokinetic properties, efficiency and low toxicity, the use of fluconazole in the prophylaxis and therapy of candidosis in organ or bone marrow transplant recipients, patients undergoing chemotherapy and AIDS patients has increased rapidly [4]. Since 1990, fluconazole-resistant isolates of $C$. albicans have been reported increasingly, particularly in AIDS patients [1]. It is now established that the prolonged use of ketoconazole and fluconazole may give rise to the emergence of C. glabrata infections [1].

The need for in-vitro susceptibility testing of clinical isolates to fluconazole has become obvious. In-vitro susceptibility testing of yeasts is affected by numerous factors such as inoculum density, media and buffer. A 
reference macro-broth dilution method (M27-P) has been proposed by the National Committee for Clinical Laboratory Standards [5]. However, this method is time-consuming, expensive and technically difficult to perform. Few commercially available kits suitable for routine clinical practice permit in-vitro susceptibility testing to fluconazole. A disk diffusion procedure (Neosensitabs; Rosco Diagnostica, Taastrup, Denmark) has been shown to be a straight-forward, low-cost and reproducible means of assessing susceptibility of yeasts [6]. The latter procedure was used in this hospital in a large-scale study to evaluate the susceptibility of a wide range of Candida isolates from patients with all types of infections. This method revealed the presence of large resistant colonies within the inhibition zones of azole compounds for almost all isolates of $C$. glabrata. The significance of these colonies was investigated in a in-vitro study.

\section{Materials and methods}

\section{Yeast isolates}

The study involved c. 3500 clinical isolates of Candida collected over a 4-year period from patients followed in different units of the teaching university hospital for all types of infections. Yeasts were identified on the basis of germ-tube formation and rice-agar-tween morphology and by their carbohydrate assimilation pattern in ID 32C test strips (bioMérieux, Marcy-l'Etoile, France). In addition, 10 isolates of C. glabrata cloned by limiting dilution and obtained from unrelated patients sampled before antifungal treatment, were included in the study.

\section{Antifungal susceptibility testing}

Disk diffusion method. Susceptibility testing was performed on Casitone (Difco) agar by a commercial agar diffusion method from Rosco Diagnostica (Taastrup, Denmark) [7]. Inoculum in sterile distilled water was prepared from fresh cultures on yeast extract-peptoneglucose (YEPD) agar containing (g/L) yeast extract (5), peptone (10), glucose (20), chloramphenicol (1) and agar (20) and standardised by haemocytometer counts. Casitone agar plates containing glucose $2 \%$ and supplemented with chloramphenicol $1 \mathrm{~g} / \mathrm{L}$ were flooded with $10 \mathrm{ml}$ of the yeast cell suspensions $(5 \times$ $10^{5}$ cells $/ \mathrm{ml}$ ). Excess fluid was removed immediately and plates were dried for $15 \mathrm{~min}$ at $37^{\circ} \mathrm{C}$. Antifungal NeoSensitabs tablets were then deposited on their surface. Following a pre-incubation period of $30 \mathrm{~min}$ at room temperature, plates were incubated for $48 \mathrm{~h}$ at $37^{\circ} \mathrm{C}$ and the diameter of each inhibition zones was measured. The presence of large colonies randomly distributed within the inhibition zones was also noted, and isolates were classified as sensitive, intermediate or resistant according to the manufacturer's recommendations.

For some experiments, susceptibility was also tested on other culture media: Oxoid HR fungal medium (Unipath, Dardilly, France), Shadomy's modified medium (yeast nitrogen base, L-asparagine, glucose, $\mathrm{NaH}_{2} \mathrm{PO}_{4}$ and $\mathrm{K}_{2} \mathrm{HPO}_{4}$ ) and a synthetic mediun from Diagnostics Pasteur (Marnes la Coquette, France).

Student's $t$ test was used for statistical evaluation of the differences in the diameters of the inhibition zones between the mutants and their parents.

Etest procedure. Five colonies from fresh cultures on YEPD agar plates were suspended in saline to achieve a turbidity comparable to a 0.5 McFarland standard. The inoculum was swabbed on to Casitone agar plates which were allowed to dry for $15 \mathrm{~min}$ before the Etest strips (AB Biodisk, Solna, Sweden) were applied. Plates were incubated at $37^{\circ} \mathrm{C}$ and MICs were read after $48 \mathrm{~h}$ as the drug concentration at which the inhibition ellipse intercepted the scale on the antifungal strips. Etest strips of fluconazole, which were stored at $-20^{\circ} \mathrm{C}$ until use, contained a concentration gradient of $0.016-256 \mathrm{mg} / \mathrm{L}$.

\section{Resistant mutants}

In the course of antifungal susceptibility testing by the disk diffusion method, one of the large resistant colonies randomly distributed within the inhibition zones was subcultured for each of the 10 strains of $C$. glabrata and each antifungal agent. These resistant mutants were then cryopreserved and compared to their parent strains by antifungal susceptibility testing, growth study and mitochondrial DNA (mtDNA) analysis.

mtDNA mutants were also induced from C. glabrata LMA 1085.90 by the use of ethidium bromide. The parent strain was cultivated for $12 \mathrm{~h}$ at $37^{\circ} \mathrm{C}$ on YEPD agar plates before a tablet of ethidium bromide (Sigma) was deposited at the centre of the plates. Plates were then incubated for $48 \mathrm{~h}$ at $37^{\circ} \mathrm{C}$ and 10 colonies growing at the margin of the ethidium bromide inhibition zone were subcultured and tested for antifungal susceptibility.

\section{Mutation stability}

In an attempt to define whether or not a reversion to the parent phenotype was possible in the absence of antifungal agent, the fluconazole-resistant mutants of strains LMA 1085.90 and LMA 5417.94 were subcultured on YEPD agar plates. One hundred bi-weekly passages were performed on YEPD agar and, after every five subcultures, the susceptibility to fluconazole was assessed by disk diffusion on Casitone agar.

\section{Cell growth}

Growth of the fluconazole-resistant mutants and of the mtDNA mutants was compared with that of the parent 
strains on yeast extract-peptone-agar plates containing glucose or glycerol $2 \%$. Growth studies were also performed in YEPD liquid medium inoculated with $10^{6}$ blastoconidia $/ \mathrm{ml}$ and incubated for $24 \mathrm{~h}$ at $37^{\circ} \mathrm{C}$ under aerobic conditions. Growth was evaluated by measuring the absorbance at $450 \mathrm{~nm}$ of a 1 in 10 dilution of the fungal suspensions. The mycelial dry weights were also determined by filtration of 5-ml samples of the fungal suspensions through $0.45-\mu \mathrm{m}$ pore size, $25-\mathrm{mm}$ diameter filters pre-weighed which were then freeze-dried. Results presented correspond to the mean (and SD) of triplicate determinations.

\section{$m t D N A$ extraction and analysis}

mtDNA was extracted as described previously [8] with slight modifications [9]. Briefly, $100 \mathrm{ml}$ of an overnight cell culture in YEPD liquid medium were pelleted by centrifugation at $500 \mathrm{~g}$ for $5 \mathrm{~min}$ at room temperature. The pellet was washed twice in sterile distilled water and resuspended in $6 \mathrm{ml}$ of a $0.5 \mathrm{M}$ sorbitol, $10 \mathrm{mM} \mathrm{Na}_{2}$ EDTA, $50 \mathrm{mM}$ Tris buffer, $\mathrm{pH} \mathrm{7.6,} \mathrm{contain-}$ ing Zymolase 20T (Seikagaku, Tokyo, Japan) $2 \mathrm{mg} / \mathrm{ml}$ and 2 -mercaptoethanol $0.2 \%$. After incubation at $37^{\circ} \mathrm{C}$ for $2 \mathrm{~h}$ with gentle shaking, and centrifugation at $1000 \mathrm{~g}$ for $10 \mathrm{~min}$ to remove nuclei, the supernate was centrifuged at $15000 \mathrm{~g}$ for $10 \mathrm{~min}$ to pellet the mitochondria. The resulting pellet was washed five times with the same buffer (without Zymolase and 2mercaptoethanol). The resulting pellet was lysed by incubation for $30 \mathrm{~min}$ at room temperature with $0.7 \mathrm{ml}$ of a $100 \mathrm{mM} \mathrm{NaCl}, 10 \mathrm{mM} \mathrm{Na}_{2}$ EDTA, sarkosyl $1 \%$, $50 \mathrm{mM}$ Tris buffer, $\mathrm{pH}$ 7.8. Nucleic acids were purified by phenol/chloroform extraction, precipitated by isopropanol and then resuspended in $10 \mathrm{mM}$ Tris buffer, pH 8, containing $1 \mathrm{mMNa}_{2}$ EDTA. RNAs were eliminated by RNAase and mtDNA was digested by Eco RV (Eurogentec, Seraing, Belgium) at $37^{\circ} \mathrm{C}$ for $2 \mathrm{~h}$ in a 10 $\mu \mathrm{l}$ volume with 1 unit of the enzyme. Finally, the digestion products were analysed by agarose $0.8 \%$ gel electrophoresis in TBE buffer ( $45 \mathrm{mMTris}, 45 \mathrm{mM}$ boric acid, $1 \mathrm{mM} \mathrm{Na}_{2}$ EDTA), stained with ethidium bromide and visualised under UV illumination.

\section{Results}

\section{Antifungal susceptibility of various Candida species}

During the experimental period, a total of 3551 clinical isolates from oropharyngeal or genital infections $(51 \%$ of the isolates) and bloodstream, organ of deep-seated infections ( $49 \%$ ) belonging to 13 different Candida species was tested for their susceptibility to antifungal agents. Applying the proposed breakpoints $(\geqslant 15$ and $20 \mathrm{~mm}$ diameter zone for polyenes and azoles, respectively, for susceptible isolates and $<10$ and $12 \mathrm{~mm}$ for resistant isolates), very few isolates were resistant to polyenes (Table 1). Among 2538 isolates of $C$. albicans, only one was moderately sensitive to ampho-

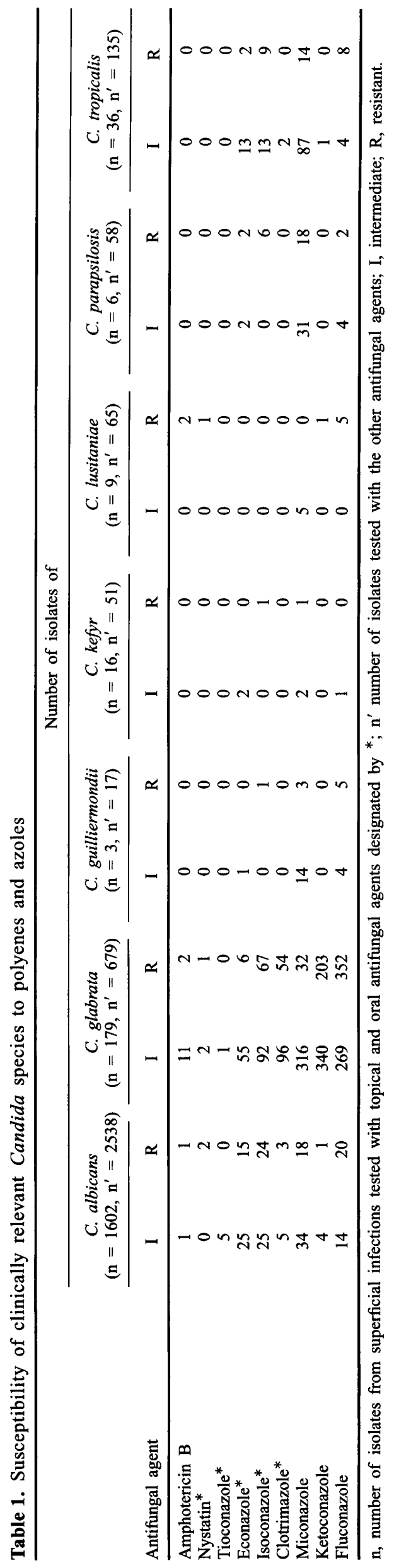


tericin B and another was completely resistant. Resistance to polyenes was also very rare in nonalbicans Candida species, and only 11 of 679 isolates of $C$. glabrata were classified as intermediate and two as resistant to amphotericin $\mathrm{B}$; two of 65 isolates of $C$. lusitaniae were resistant.

Resistance to azoles was relatively uncommon. Fourteen and $20 \mathrm{C}$. albicans isolates were classified as intermediate or resistant to fluconazole, respectively (Table 1). Resistant yeasts included isolates of $C$. lusitaniae, C. parapsilosis, C. kefyr, C. guilliermondii and $C$. tropicalis (Table 1). No resistance was observed in the other species included in this study, i.e., C. famata (one isolate), C. globosa (one), C, humicola (one), C. lipolytica (one), C. pelliculosa (three) and $C$. rugosa (one), and all the isolates were sensitive to tioconazole. Conversely, resistance to azoles was particularly frequent for $C$. glabrata: $52 \%$ of the isolates tested were classified as resistant to fluconazole and $39 \%$ as intermediate (Table 1). However, only 15 of these isolates, all from patients who had received fluconazole in a 3-month period before the isolation of the yeast, were completely resistant to the drug. The other isolates presented welldefined inhibition zones in which randomly distributed large colonies were observed, some growing directly in contact with the disk. These colonies, which led to classification of the isolate as intermediate or resistant according to their distance from the disk, suggested the presence of resistant mutants.

\section{Susceptibility pattern of $C$. glabrata strains}

To confirm this hypothesis, 10 isolates of $C$. glabrata from unrelated patients were cloned by limiting dilution
Table 2. Antifungal susceptibility patterns of $C$. glabrata azole-resistant mutants and their parent strains

\begin{tabular}{|c|c|c|}
\hline \multirow[b]{2}{*}{$\begin{array}{l}\text { Antifungal } \\
\text { agent }\end{array}$} & \multicolumn{2}{|c|}{$\begin{array}{l}\text { Mean (SD) diameter of the inhibition zones } \\
(\mathrm{mm})\end{array}$} \\
\hline & $\begin{array}{l}\text { Parent strains } \\
\quad(\mathrm{n}=10)\end{array}$ & $\begin{array}{l}\text { Mutants } \\
(\mathrm{n}=58)\end{array}$ \\
\hline Amphotericin B & $27.4 \quad(1.6)$ & $33.1(2.6)$ \\
\hline Nystatin & $28.9 \quad(3.2)$ & $32.9(2.4)$ \\
\hline Tioconazole & $31.6 \quad(5.7)$ & $34 \quad(5.3)$ \\
\hline Econazole & $24.1^{*}(3.8)$ & $\begin{array}{c}15.2(3.4) \\
\text { (R for } 31 \text { mutants) }\end{array}$ \\
\hline Miconazole & $24^{*} \quad(1.5)$ & $\begin{array}{c}16.5(3.7) \\
\text { ( } \mathrm{R} \text { for } 35 \text { mutants) }\end{array}$ \\
\hline Isoconazole & $20.9^{*}(1.7)$ & $\mathrm{R}$ \\
\hline Clotrimazole & $28.2^{*}(3.6)$ & $\mathrm{R}$ \\
\hline Ketoconazole & $29.6^{*}(1.6)$ & $\mathrm{R}$ \\
\hline Fluconazole & $27.1^{*}(2.5)$ & $\mathrm{R}$ \\
\hline
\end{tabular}

$\mathrm{n}$, number of parent strains or mutants analysed; ${ }^{*}$, presence of large colonies randomly distributed within the inhibition zone and therefore considered as resistant mutants, except for strain LMA 5574.94 with econazole and miconazole; $\mathrm{R}$, complete resistance with an homogeneous growth directly in contact with the antifungal disk.

and their susceptibility to polyenes and azoles was studied by the disk diffusion method in Casitone agar; results are presented in Table 2 and Fig. 1. Large colonies randomly distributed within the inhibition zones, therefore corresponding to resistant mutants, were observed after incubation for $48 \mathrm{~h}$ for all the strains tested with isoconazole, clotrimazole, ketoconazole and fluconazole, and for all except one with econazole and miconazole. For each strain and each antifungal agent, the resistant colony nearest the disk was subcultured. Fifty-eight mutants were subcultured and their susceptibility to polyenes and azoles was determined and compared to that of the parent strains. The mutants exhibited the same susceptibility pattern regardless of which antifungal agent allowed their

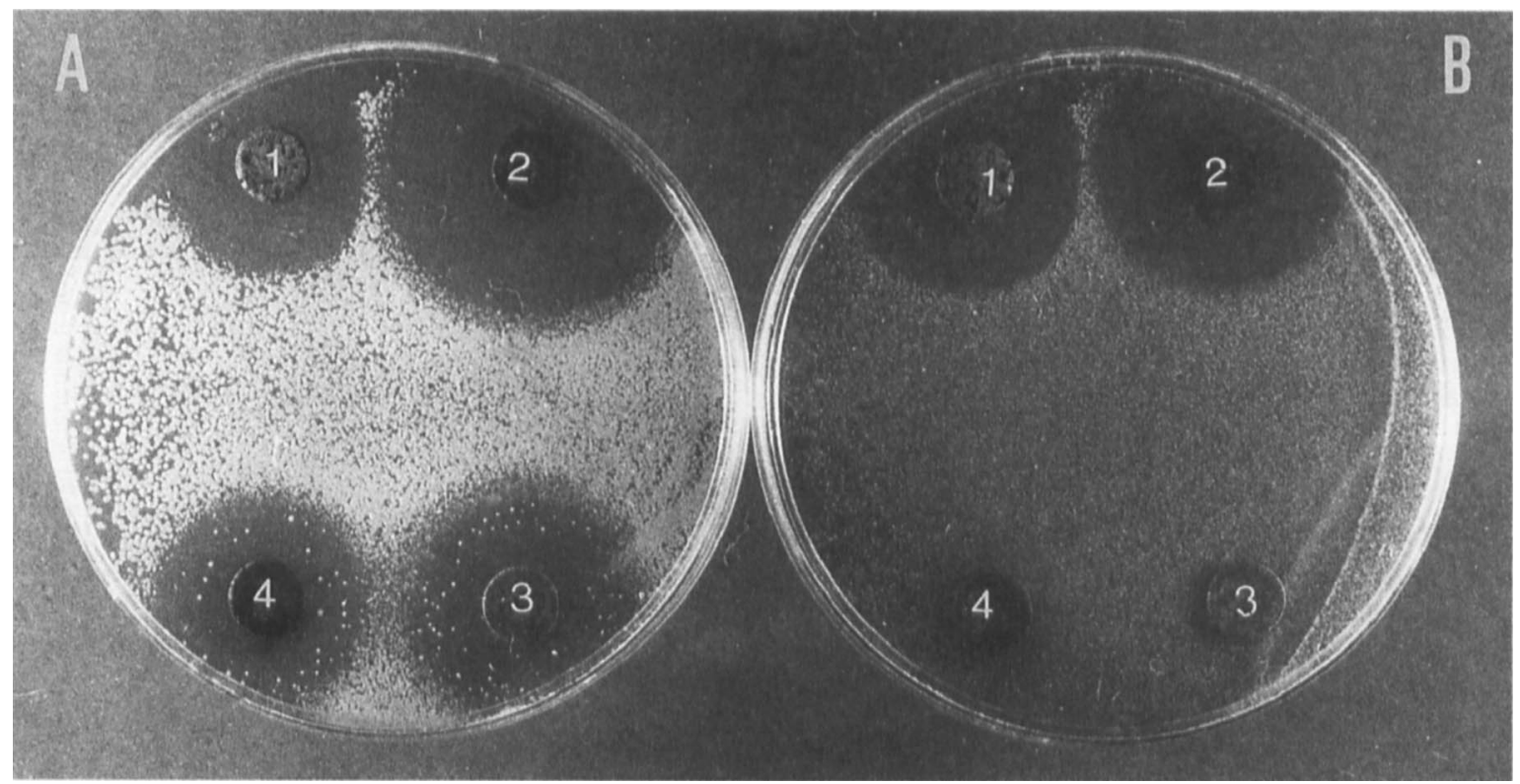

Fig. 1. Antifungal susceptibility testing by the disk diffusion method. Strain LMA 1085.90 (A) and its fluconazoleresistant mutant (B) were inoculated on Casitone agar. Tablets of amphotericin B (1), tioconazole (2), ketoconazole (3) and fluconazole (4) were deposited on the surface of the agar plates and plates were incubated for $48 \mathrm{~h}$ at $37^{\circ} \mathrm{C}$. 
selection, showing a cross-resistance to all the azoles tested except tioconazole. All mutants were completely resistant to isoconazole, clotrimazole, ketoconazole and fluconazole. No inhibition zone was seen with econazole and miconazole for 31 and 35 of the mutants, respectively, whereas the other mutants exhibited a marked decrease in their sensitivity. Conversely, the mutants remained susceptible to tioconazole. Moreover, a significant increase in the susceptibility to polyenes was noted, the diameter of the inhibition zones being statistically greater for the mutants than for the parent strains $(\mathrm{t}=6.83 ; \mathrm{df}=66 ; \mathrm{p}<0.001$ for amphotericin $\mathrm{B}$ and $t=4.66 ; \mathrm{df}=66 ; \mathrm{p}<0.001$ for nystatin).

\section{Influence of the culture medium}

Neither mutants nor parent strains grew on HR medium. Furthermore, no resistant colonies were observed from the parent strains on Shadomy's medium or on synthetic medium. However, the mutants previously selected on Casitone agar were completely resistant to econazole, isoconazole, clotrimazole, ketoconazole and fluconazole on these two culture media. Similarly, an increase in the diameter of the inhibition zones was noted with tioconazole and polyenes for the mutants compared with the parent strains.

\section{Determination of fuconazole MICs by the Etest procedure}

The Etest procedure performed on Casitone agar confirmed the results obtained by the disk diffusion method, demonstrating fluconazole MICs of 24$64 \mathrm{mg} / \mathrm{L}$ for the parent strains (median $32 \mathrm{mg} / \mathrm{L}$ ), but $>252 \mathrm{mg} / \mathrm{L}$ for the fluconazole-resistant mutants.

\section{Stability of the phenotype}

The fluconazole-resistant mutants of strains LMA 90.1085 and LMA 94.5417 were subcultured on YEPD agar and resistance to fluconazole was monitored by the disk diffusion method on Casitone agar every five passages. After 100 generations, the mutants remained completely resistant to the drug.

\section{Growth studies}

Comparative growth studies on agar medium containing glucose or glycerol $2 \%$ revealed the respiratory deficiency of the fluconazole-resistant mutants. The mutants produced small colonies on glucose-containing agar and did not grown in the presence of glycerol $2 \%$ as the sole carbon source (Table 3 ). Conversely, culture of the parent strains yielded large colonies on glucoseagar plates, and growth was only slightly affected by the presence of glycerol.

Growth studies in YEPD liquid medium confirmed the respiratory deficiency. Growth of the mutants was markedly reduced compared with the parent strains; differences in optical densities of 24-h-old cultures between the mutants and their parents ranged from $28 \%$ to $49 \%$ (Table 3 ). Likewise, the mycelial dry weights of the mutants varied from $26 \%$ to $37 \%$ of those obtained for the parent strains (data not shown).

\section{Analysis of $m t D N A$}

Restriction endonuclease analysis of mtDNA was performed with Eco RV, which has been shown to be the most discriminatory endonuclease for polymorphism analysis in $C$. glabrata [9]. Three different electrophoretic patterns were observed in parent strains (Fig. 2). Pattern A (Fig. 2, lines 4, 8, 14, 17 and 22) was the most common ( 5 of the 10 strains) and showed two restriction fragments of $c .20$ and $5 \mathrm{~kb}$. An additional restriction site was seen in patterns $B$ and $C$ resulting in three fragments of c. 20,5 and $1.7 \mathrm{~kb}$ (Fig. 2, lines 6, 12, and 20) and 20, 3.5 and $1.7 \mathrm{~kb}$ (Fig. 2 , lines 2 and 10) respectively.

Electrophoresis revealed important deletions of the mtDNA in fluconazole-resistant mutants. No mtDNA was detected after ethidium bromide staining for six of the mutants. For three other mutants, the banding pattern consisted of a unique band with a mol. wt that was always lower than those detected in parent strains (Fig. 2, lines 11, 13 and 15). Interestingly, one mutant exhibited a very intense band lower than $0.8 \mathrm{~kb}$ (Fig. 2 , line 11), suggesting a repeated short sequence

Table 3. Comparative growth study of the fluconazole-resistant mutants and their parent strains

\begin{tabular}{|c|c|c|c|c|c|c|}
\hline \multirow[b]{2}{*}{ Strain } & \multicolumn{2}{|c|}{ Colonies on glucose-agar } & \multicolumn{2}{|c|}{ Growth on glycerol-agar } & \multicolumn{2}{|c|}{ Mean (SD) growth in YEPD liquid medium* } \\
\hline & WT & M & WT & $\mathrm{M}$ & WT & M \\
\hline LMA 1085.90 & Large & Small & + & - & $1.079(0.014)$ & $0.405(0.013)$ \\
\hline LMA 5756.90 & Large & Small & + & - & $0.904(0.035)$ & $0.281(0.007)$ \\
\hline LMA 883.91 & Large & Small & + & - & $1.032(0.020)$ & $0.314(0.018)$ \\
\hline LMA 970.91 & Large & Small & + & - & $1.066(0.015)$ & $0.344(0.037)$ \\
\hline LMA 1614.91 & Large & Small & + & - & $0.848(0.037)$ & $0.414(0.013)$ \\
\hline LMA 1456.92 & Large & Small & + & - & $1.095(0.043)$ & $0.310(0.006)$ \\
\hline LMA 5338.94 & Large & Small & + & - & $0.906(0.032)$ & $0.332(0.015)$ \\
\hline LMA 5417.94 & Large & Small & + & - & $0.988(0.022)$ & $0.361(0.014)$ \\
\hline LMA 5574.94 & Large & Small & + & - & $1.030(0.023)$ & $0.353(0.015)$ \\
\hline LMA 5579.94 & Large & Small & + & - & $1.006(0.009)$ & $0.360(0.012)$ \\
\hline
\end{tabular}

WT, parent wild-type strain; M, fluconazole-resistant mutant. ${ }^{*}$ Growth in YEPD liquid medium was evaluated after incubation for $24 \mathrm{~h}$ at $37^{\circ} \mathrm{C}$ by measuring the $A_{450}$ of a 1 in 10 - dilution of the fungal suspensions. 


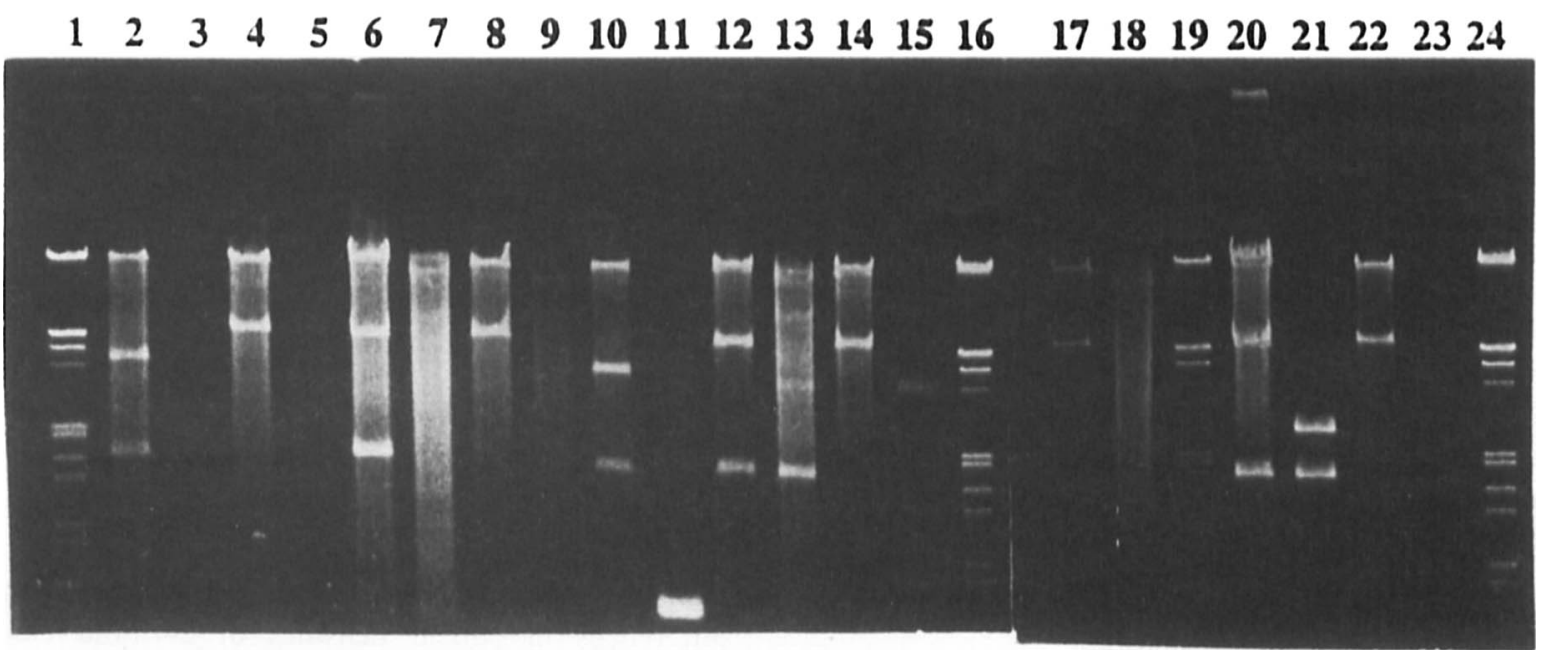

Fig. 2. Electrophoretic patterns of the 10 parent wild-type strains and their fluconazole-resistant mutants obtained by EcoRV digestion of mtDNA. Lanes 1, 16, 19 and 24, $\lambda$ double-digest HindIII EcoRI; 2, strain LMA 5756.90; 4, LMA $883.91 ; 6$, LMA $1614.91 ; 8$, LMA 1456.92; 10, LMA 5338.94; 12, LMA 5417.94; 14, LMA 5574.94; 17, LMA 5579.94; 20, LMA $970.91 ; 22$, LMA 1085.90 and their respective fluconazole-resistant mutants (lanes 3, 5, 7, 9, 11, $13,15,18,21,23)$

comparable to those observed in 'petite' mutants of Saccharomyces cerevisiae [10]. The last mutant, corresponding to strain LMA 970.91, presented two mol.wt fragments, one equivalent to the $1.7-\mathrm{kb}$ fragment of the parent strain, whereas the other, slightly greater in size, did not correspond to any of the fragments detected in the parent strain (Fig. 2, line 21). No relationship was observed between the type of mutation (total or partial deletion of the mtDNA) and the banding pattern ( $\mathrm{A}, \mathrm{B}$ or $\mathrm{C}$ ) of the parent strains.

\section{Study of ethidium bromide-induced mutants}

The 10 respiratory mutants induced by ethidium bromide treatment of the wild-type strain LMA 1085.90 were unable to grow on glycerol-agar plates and appeared to be completely devoid of mtDNA after electrophoretic migration (Fig. 3, lines 4-9). In addition, antifungal susceptibility studies showed that they were all sensitive to polyenes and tioconazole and

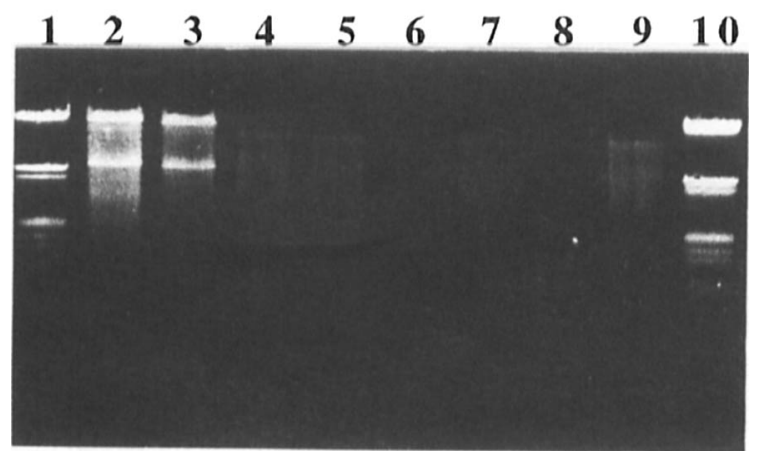

Fig. 3. Electrophoretic patterns of ethidium bromideinduced mutants of strain LMA 1085.90 (lanes 4-9) and of colonies isolated far from the ethidium bromide inhibition zone (lanes 2 and 3) obtained by EcoRV digestion of mtDNA. Mol. wt markers ( $\lambda$ double-digest HindIII EcoRI) are shown in lanes 1 and 10. completely resistant to the other azole compounds. Conversely, colonies isolated far from the ethidium bromide inhibition zone preserved their ability to grow on glycerol-agar and exhibited an Eco RV electrophoretic pattern (Fig. 3, lines 2 and 3) and a susceptibility pattern identical to that of the parent strain.

\section{Discussion}

The agar diffusion method used in this study showed that, despite the widespread use of antifungal agents, resistance to azoles remains uncommon in most Candida species; $<2 \%$ of $C$. albicans isolates were intermediate or resistant to fluconazole. The situation was different for $C$. glabrata, a high proportion of isolates were intermediate or resistant to azoles, particularly the less hydrophobic ketoconazole and fluconazole. With c. $52 \%$ of the isolates resistant to fluconazole, these results are in agreement with those of workers who used other experimental procedures, including the reference macro-broth dilution procedure [11-13]. However, only 15 of the 679 isolates tested were completely resistant to fluconazole. More frequently, large colonies randomly distributed within the inhibition zones were observed which suggested a mutation leading to azole resistance and posed the question of the mechanisms of this acquired resistance.

The target of azoles is clearly identified as the cytochrome P-450-dependent 14 $\alpha$-sterol demethylase (P-450DM), but the mechanisms of acquired resistance to these antifungal agents are not understood completely. Several resistance mechanisms have been described in $C$. albicans $[14,15]$, particularly nonspecific mechanisms such as enhanced efflux of drug involving ATP binding cassettes or major facilitators, 
responsible for multidrug resistance [16-18]. Also, more specific mechanisms consisting of changes in the binding site [19] or catalytic site [20] on the azole target, amplification or increasing transcription of the P-450DM gene $[16,21,22]$ or $\Delta 5-6$ sterol desaturase mutations $[14,23]$ have been reported. Conversely, relatively few and sometimes conflicting data are available concerning the resistance mechanisms in $C$. glabrata. The more significant studies in this field were performed by Hitchcock et al. [24-26] and Vanden Bossche et al. [14, 27, 28]. These studies relied on the comparison of two clinical isolates obtained from a unique patient in which the microorganism had become resistant during treatment with fluconazole. The resistance to fluconazole - which was associated with resistance to ketoconazole and itraconazole - seemed to be due to a failure of intracellular accumulation of drug related to enhanced efflux [24-28] and associated with an increase in sterol biosynthesis [27]. Moreover, RFLP analysis of the genomic DNA of the two isolates before and after treatment showed that they were not related, suggesting selection by the drug of a resistant strain within a double population of yeasts [24].

The present study, performed with 10 strains cloned by limiting dilution and obtained from unrelated patients, clearly indicates that resistance to azoles for C. glabrata may be acquired by mutation. Antifungal susceptibility testing performed on Casitone agar revealed the presence of large resistant colonies randomly distributed within the inhibition zones for all the azoles tested except tioconazole. The susceptibility pattern of these colonies to polyenes and azoles (determined by the same method) was constant, regardless of the antifungal agent allowing selection. Moreover, colonies selected on the basis of resistance to fluconazole remained resistant to the drug after multiple subcultures in the absence of the antifungal agent, demonstrating stability of the phenotype.

Results obtained on Casitone agar for the fluconazoleresistant mutants were confirmed with Shadomy's modified medium and a synthetic medium, and by determination of fluconazole MICs by the Etest procedure, a method which has been shown to give good agreement with the macro-broth reference dilution method [29-31]. Fluconazole MICs ranged from 24 to $64 \mathrm{mg} / \mathrm{L}$ for the parent strains, consistent with previous reports indicating that this yeast species is relatively resistant to fluconazole [32, 33].

For most clinical isolates classified as intermediate or resistant, resistant colonies were detected within the inhibition zones only after incubation for $48 \mathrm{~h}$. Therefore, a comparative study of the growth of the fluconazole-resistant mutants and their parent strains was performed which revealed the respiratory deficiency of the mutants. RFLP analysis of their mtDNA confirmed the mitochondrial origin of this respiratory deficiency. Six of the mutants exhibited a complete loss of the mtDNA, analogous to rho ${ }^{0}$ 'petite' mutants of $S$. cerevisiae, whereas four other mutants showed partial deletions of the mtDNA comparable to 'petite' rho- of $S$. cerevisiae [34]. In addition, the induction of the complete loss of mtDNA by ethidium bromide treatment of a respiratory competent strain suggested that this resistance was a consequence of the respiratory deficiency. It is now well established that the synthesis of P-450DM is stimulated under anaerobic conditions or by the presence of a high concentration of glucose (Crabtree effect) [35]. Similarly, in C. albicans, anaerobic conditions inhibit the antifungal activity of ketoconazole [36]. Therefore, it can be speculated that the absence of respiration imposed by culture conditions (anaerobic conditions, catabolic repression, respiratory inhibitors) or by spontaneous or induced mutations, may stimulate the biosynthesis of P-450DM, leading to the resistance of the yeast to azole derivatives.

The results of the present study confirm and extend previous reports of the high incidence of fluconazole resistance on $C$. glabrata. The clinical failure of fluconazole therapy in C. glabrata infections has been related to the lower susceptibility of this yeast species to azole derivatives [1], but another hypothesis would be the selection in vivo of mutants resistant to the drug. The clinical relevance of the resistance mechanism identified here remains to be defined. It is possible that in the presence of azoles these mutants present a selective advantage (via the over-expression of P450-DM) allowing them to develop instead of the parent cells. However, during the course of this study, the recovery of an isolate of $C$. glabrata presenting with a complete resistance to azoles remained very rare. Therefore, it seems possible that the selection of azole-resistant mtDNA mutants rarely occurs in vivo, or that this mutation leads to a reduced virulence. In C. albicans, alterations of hyphal growth [37] and loss of virulence [38] associated with acquired resistance to azoles have been described. Several authors have reported an increased incidence of oro-intestinal colonisation by $C$. glabrata in bone marrow transplant recipients undergoing prophylactic fluconazole or ketoconazole therapy [39, 40], without a concomittant increase in disseminated infections. Nevertheless, these mutants which reveal differences in their susceptibility to azoles (tioconazole versus others), provide an interesting model to study the mechanism of the action of azole derivatives.

We sincerely thank Professor Francis Karst (University of Poitiers) for helpful discussion.

\section{References}

1. Odds FC. Epidemiological shifts in opportunistic and nosocomial Candida infections: mycological aspects. Int $J$ Antimicrob Agents 1996; 6: 141-144. 
2. Nguyen $\mathrm{MH}$, Peacock JE, Morris $\mathrm{AJ}$ et al. The changing face of candidemia: emergence of non-Candida albicans species and antifungal resistance. Am J Med 1996; 100: 617-623.

3. Price MF, Larocco MT, Gentry LO. Fluconazole susceptibilities of Candida species and distribution of species recovered from blood cultures over a 5-year period. Antimicrob Agents Chemother 1994; 38: 1422-1424.

4. Bodey GP. Azole antifungal agents. Clin Infect Dis 1992; 14 Suppl 1: S161-S169.

5. National Committee for Clinical Laboratory Standards. Reference method for broth dilution antifungal susceptibility testing of yeasts. Proposed guideline M-27P. Villanova, PA, National Committee for Clinical Laboratory Standards. 1992.

6. Sandven P, Bjørneklett A, Maeland A and the Norwegian Yeast Study Group. Susceptibilities of Norwegian Candida albicans strains to fluconazole: emergence of resistance. Antimicrob Agents Chemother 1993; 37: 2443-2448.

7. Casals JB. Tablet sensitivity testing of pathogenic fungi. J Clin Pathol 1979; 32: 719-722.

8. Defontaine A, Lecocq FM, Hallet JN. A rapid miniprep method for the preparation of yeast mitochondrial DNA. Nucleic Acids Res 1991; 19: 185.

9. Defontaine A, Coarer M, Bouchara JP. Contribution of various techniques of molecular analysis to strain identification of Candida glabrata. Microb Ecol Health Dis 1996; 9: 27-33.

10. Locker J, Lewin A, Rabinowitz $M$. The structure and organization of mitochondrial DNA from petite yeast. Plasmid 1979; 2: 155-181.

11. Stevens DA, Stevens JA. Cross-resistance phenotypes of fluconazole-resistant Candida species: results with 655 clinical isolates with different methods. Diagn Microbiol Infect Dis 1996; 26: 145-148.

12. May JL, King A, Warren CA. Fluconazole disc diffusion testing from the routine laboratory. $J$ Antimicrob Chemother 1997; 40: $511-516$

13. Bille J, Glauser MP and the Fluconazole Global Susceptibility Study Group. Evaluation of the susceptibility of pathogenic Candida species to fluconazole. Eur J Clin Microbiol Infect Dis 1997; 16: 924-928.

14. Vanden Bossche H, Marichal P, Odds FC. Molecular mechanisms of drug resistance in fungi. Trends Microbiol 1994; 2: 393-400.

15. White TC, Marr KA, Bowden RA. Clinical, cellular, and molecular factors that contribute to antifungal drug resistance. Clin Microbiol Rev 1998; 11: 382-402.

16. Sanglard D, Kuchler K, Ischer F, Pagani J-L, Monod M, Bille J. Mechanisms of resistance to azole antifungal agents in Candida albicans isolates from AIDS patients involve specific multidrug transporters. Antimicrob Agents Chemother 1995; 39: $2378-2386$

17. Goldway M, Teff D, Schmidt R, Oppenheim AB, Koltin Y. Multidrug resistance in Candida albicans: disruption of the $B E N^{R}$ gene. Antimicrob Agents Chemother 1995; 39: 422-426.

18. Prasad R, De Wergifosse P, Goffeau A, Balzi E. Molecular cloning and characterization of a novel gene of Candida albicans, $C D R 1$, conferring multiple resistance to drugs and antifungals. Curr Genet 1995; 27: 320-329.

19. Joseph-Horne T, Hollomon D, Loeffler RST, Kelly SL. Altered P450 activity associated with direct selection for fungal azole resistance. FEBS Lett 1995; 374: 174-178.

20. Bard M, Lees ND, Barbuch RJ, Sanglard D. Characterization of a cytochrome P450 deficient mutant of Candida albicans. Biochem Biophys Res Commun 1987; 147: 794-800.

21. Albertson GD, Niimi M, Cannon RD, Jenkinson HF. Multiple efflux mechanisms are involved in Candida albicans fluconazole resistance. Antimicrob Agents Chemother 1996; 40: 2835-2841.

22. White TC. Increased mRNA levels of ERG16, CDR, and $M D R 1$ correlate with increases in azole resistance in Candida albicans isolates from a patient infected with human immunodeficiency virus. Antimicrob Agents Chemother 1997; 41: $1482-1487$.

23. Hitchcock CA. Resistance of Candida albicans to azole antifungal agents. Biochem Soc Trans 1993; 21: 1039-1047.

24. Hitchcock CA, Pye GW, Troke PF, Johnson EM, Warnock DW. Fluconazole resistance in Candida glabrata. Antimicrob Agents Chemother 1993; 37: 1962-1965.

25. Parkinson T, Falconer DJ, Hitchcock CA. Fluconazole resistance due to energy-dependent drug efflux in Candida glabrata. Antimicrob Agents Chemother 1995; 39: 1696-1699.

26. Clark FS, Parkinson T, Hitchcock CA, Gow NAR. Correlation between rhodamine 123 accumulation and azole sensitivity in Candida species - possible role for drug efflux in drug resistance. Antimicrob Agents Chemother 1996; 40: 419-425.

27. Vanden Bossche H, Marichal P, Odds FC, Le Jeune L, Coene M-C. Characterization of an azole-resistant Candida glabrata isolate. Antimicrob Agents Chemother 1992; 36: 2602-2610.

28. Marichal P, Vanden Bossche H, Odds FC et al. Molecular biological characterization of an azole-resistant Candida glabrata isolate. Antimicrob Agents Chemother 1997; 41: 2229-2237.

29. Barry AL, Brown SD. Fluconazole disk diffusion procedure for determining susceptibility of Candida species. J Clin Microbiol 1996; 34: 2154-2157.

30. Wanger A, Mills K, Nelson PW, Rex JH. Comparison of Etest and National Committee for Clinical Laboratory Standards broth macrodilution method for antifungal susceptibility testing: enhanced ability to detect amphotericin B-resistant Candida isolates. Antimicrob Agents Chemother 1995; 39: 2520-2522.

31. Colombo AL, Barchiesi F, McGough DA, Rinaldi MG. Comparison of Etest and National Committee for Clinical Laboratory Standards broth macrodilution method for azole antifungal susceptibility testing. J Clin Microbiol 1995; 33: $535-540$.

32. Pfaller MA, Barry AL. In vitro susceptibilities of clinical yeast isolates to three antifungal agents determined by the microdilution method. Mycopathologia 1995; 130: 3-9.

33. Cormican MG, Hollis RJ, Pfaller MA. DNA macrorestriction profiles and antifungal susceptibility of Candida (Torulopsis) glabrata. Diagn Microbiol Infect Dis 1996; 25: 83-87.

34. Slonimski PP, Perrodin G, Croft JH. Ethidium bromide induced mutation of yeast mitochondria: complete transformation of cells into respiratory deficient non-chromosomal 'petites'. Biochem Biophys Res Commun 1968; 30: 232-239.

35. Wiseman A, King DJ. Microbial oxygenases and their potential application. In: Wiseman A (ed) Topics in enzyme and fermentation biotechnology, vol 6. Chichester, Ellis Horwood. 1982: $151-206$.

36. Uno J, Shigematsu ML, Araï T. Primary site of action of ketoconazole on Candida albicans. Antimicrob Agents Chemother 1982; 21: 912-918.

37. Lees ND, Broughton MC, Sanglard D, Bard M. Azole susceptibility and hyphal formation in a cytochrome P-450deficient mutant of Candida albicans. Antimicrob Agents Chemother 1990; 34: 831-836.

38. Smith KJ, Warnock DW, Kennedy CTC et al. Azole resistance in Candida albicans. J Med Vet Mycol 1986; 24: 133-144.

39. Goodman JL, Winston DL, Greenfield RA et al. A controlled trial of fluconazole to prevent fungal infections in patients undergoing bone marrow transplantation. $N$ Engl J Med 1992; 326: $845-851$

40. Shepp DH, Klosterman A, Siegel MS, Meyers JD. Comparative trial of ketoconazole and nystatin for prevention of fungal infection in neutropenic patients treated in a protective environment. $J$ Infect Dis 1985; 152: 1257-1263. 